\title{
The value of Gleason score and prostate-specific antigen level in predicting the need for a baseline nuclear bone scan in patients with newly diagnosed 84 prostate cancer cases
}

\author{
Hind Chorfi, Nadia Ismaili Alaoui \\ Department of Nuclear Medicine, University Hospital Hassan II, FES, Morocco
}

[Presented at the $4^{\text {th }}$ African Regional IRPA congress (AFRIRPA04), which was held from September 13-17, 2014 in Rabat, Morocco. This paper was reviewed and accepted by the scientific committee of the $4^{\text {th }}$ African Regional IRPA congress]

\section{Conference Proceeding}

\begin{abstract}
Purpose: The objective of the present study was to correlate the prostate-specific antigen (PSA) level and Gleason score with the baseline bone scan results in patients with newly diagnosed prostate cancer and try to determine a group of patients whose risk of bone metastases is low enough to omit safely this staging modality. Methods: This retrospective study included 84 consecutive patients with newly diagnosed prostate cancer (Pca) who underwent a staging bone scan in Nuclear Medicine department between August 2013 and August 2014. Data were collected on age, bony pain, prostate-specific antigen (PSA) level and Gleason score, then, bone scan results were analyzed with respect to these parameters. Bone scan was recorded as positive, negative or equivocal. In case of equivocal lesions, a single-photon emission computed tomography combined with computed tomography (SPECT-CT) was performed allowing a better morphological precision. Results: The median age of the patients was 71, 38 years. Bone metastases were detected in 41 patients (49\% of cases), bony pain was a reliable presenting sign of skeletal involvement. Both prostate-specific antigen (PSA) level and Gleason score were independent predictors of positive bone scan. However, the combination of these two parameters enhanced predictability of bone scan results. According to this study, the risk to develop a bone metastasis was very low in asymptomatic patients with PSA level $<20 \mathrm{ng} / \mathrm{ml}$ irrespective of the Gleason score or with PSA level $<30 \mathrm{ng} / \mathrm{ml}$ associated to a Gleason score $<7$. Conclusion: The present study discourages the routine use of bone scan as a pre-treatment staging modality in asymptomatic patients with PSA level $<20 \mathrm{ng} / \mathrm{ml}$ irrespective of the Gleason score or with PSA level $<30 \mathrm{ng} / \mathrm{ml}$ associated to a Gleason score $<7$, allowing considerable cost savings and decreasing time from diagnosis to treatment.
\end{abstract}

Keywords: Prostate Cancer; PSA level; Gleason Score; Bone Scan

\section{Introduction}

Prostate cancer is considered currently the most common malignancy and the second leading cause of cancer death among men aged over 50 years in developed countries. Although it is one of the few cancers that grow so slowly that it may never be life threatening, it can show an aggressive pattern that may spread and cause the death of patients mainly due to malignant involvement of bone. ${ }^{1,2}$ Therefore, early diagnosis of metastatic bone involvement in prostate cancer is crucial for selecting appropriate therapy, to assess the patient's prognosis, and to evaluate the efficacy of bone-specific treatments that may reduce future bone associated morbidity. ${ }^{1}$

Historically, nuclear bone scan is the investigation of choice to evaluate bone metastases. It has a great sensitivity; however, it lacks specificity prompting the need for further im- aging that, in turn, create anxiety for patients, add considerable cost, and delay therapy. ${ }^{3}$

The advent and the development of different techniques for measurement of prostate specific antigen (PSA) level since the 1990s has led to spectacular changes in the incidence, age and cancer stage at diagnosis. Following its introduction, the test soon became the most commonly used screening method for the diagnosis and follow-up in the management of prostate cancer patients. ${ }^{1,3}$ More recent studies demonstrated Gleason grade to be an independent predictor for positive bone scan and that its utilization may avoid a considerable number of bone scans. ${ }^{4}$

International guidelines uniformly suggest no routine staging for bone metastases in low-risk prostate cancer allowing more selectivity in performing bone scans. ${ }^{5}$ The European Associa- 
tion of Urology (EAU) guidelines, updated in April 2014, state that bone scan is recommended in asymptomatic patients only if the PSA level $>10 \mathrm{ng} / \mathrm{mL}$ or Gleason score $\geq 8$ or clinical stage $\geq \mathrm{T} 3$ (intermediate-/high-risk situations). It should also be obtained in symptomatic patients, independently of the PSA level, Gleason score or clinical stage. ${ }^{6}$

This study was conducted to correlate bone scan results with prostate-specific antigen level and Gleason score in patients with newly diagnosed prostate cancer with the main aim of identifying a group of patients with a low probability of bone metastases who did not require a pretreatment nuclear bone scan, and assessing the safety of implementing the EAU guidelines in our patients.

\section{Methods and Materials}

\section{Patients}

This is a retrospective and analytic study reviewing 84 consecutive patients with newly diagnosed prostate cancer referred to Nuclear Medicine department to undergo a staging bone scan between August 2013 and August 2014. All patients with known Gleason Score and with PSA level measured in absence of treatment were included. Data were gathered on age, bony pain, PSA level, Gleason score and associated bone scan findings.

\section{Bone scan}

Bone scintigraphy was carried out 2-4 h after an intravenous injection of $740 \mathrm{MBq}$ of $99 \mathrm{mTc}$-methylene diphosphonate. Planar images were acquired on a hybrid SPECT-CT dual head Gamma camera (SYMBIA T6, Siemens Medical Solutions). Anterior and posterior whole body planar images were acquired in a continuous mode at a speed of $15 \mathrm{~cm} / \mathrm{min}$; using parallel-hole, low-energy, high resolution collimators, with the patient in the supine position. Immediately after acquisition, the planar images were evaluated by a nuclear medicine consultant to decide the need for an additional imaging in the form of SPECT or SPECT-CT.

SPECT was done only for the volume defined based on planar bone scan. The acquisition orbits were body contour orbits over $360^{\circ}$ arcs, with the use of 64 stops. For 64 stops, emission data were acquired for $30 \mathrm{~s}$ per stop. The image acquisition matrix was $128 \times 128$. SPECT was followed by CT examination with acquisition parameters of $130 \mathrm{kV}, 100 \mathrm{mAs}$, using standard filters and slice thickness of $2 \mathrm{~mm}$.

\section{Results}

The median age of our patients was 71, 38 years $(50-92$ years). The most affected age group was between 70 and 80 years (49\% of all patients) Figure 1.

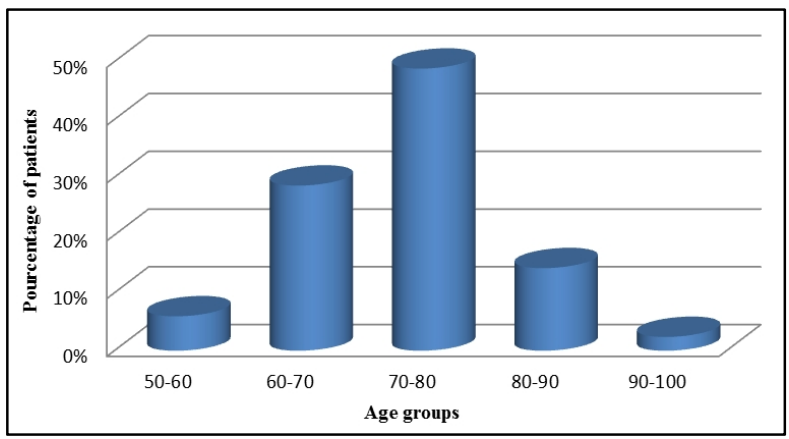

FIG. 1: Distribution of patients according to their age group.

All patients included in our study had a prostate adenocarcinoma, the other histological types including urothelial carcinoma, sarcoma or lymphoma have not been found.

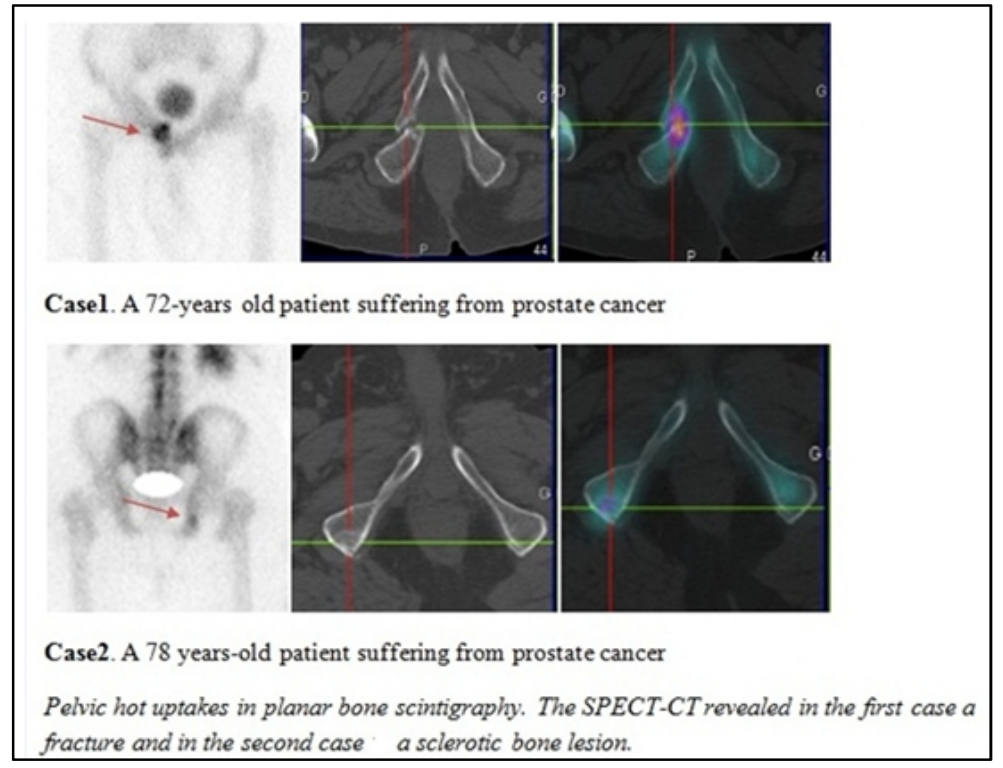

FIG. 2: Contribution of the SPECT_CT in differentiating between a benign and a malignant condition. 
Bone scan findings were tentatively classified into three categories, positive in 35 patients, negative in 26 patients and equivocal in 23 patients. SPECT CT was performed in patients who had indeterminate lesions on planar bone scintigraphy; $74 \%$ of these lesions were rated as benign and $26 \%$ as malignant, predominantly sclerotic in $83 \%$ of cases and lytic in $17 \%$ of cases in computed tomography images (Figure 2). Accordingly, the overall proportion of positive bone scans was $49 \%(41 / 84)$.

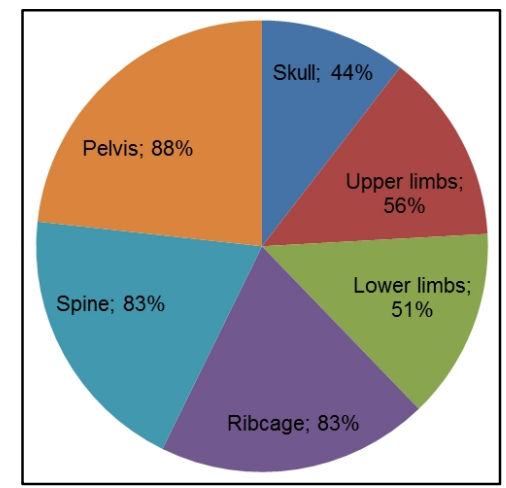

FIG. 3: Predilection sites of bone metastases.

The predilection sites of bone metastases were:

- $\quad$ Pelvis, the most commonly involved (88\%),

- $\quad$ Spine $(83 \%)$,

- $\quad$ Ribcage (83\%),

- Upper limbs (56\%),

- Lower limbs (51\%),

- $\quad$ Skull, the less involved (44\%) (Figure 3).

Bone metastases were multiple in $88 \%$ of cases against only $12 \%$ of solitary lesions.

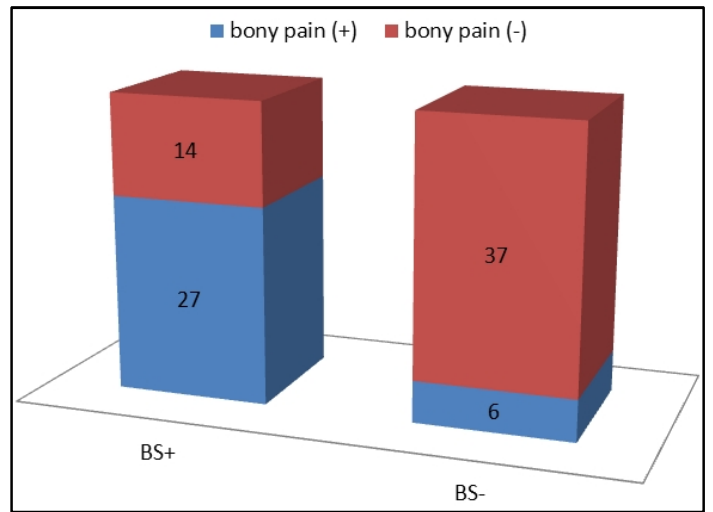

FIG. 4: Skeletal symptoms in positive and negative bone scans (BS).

The presence of skeletal symptoms was definitely a reliable presenting sign of bone involvement (Figure 4). Table 1 shows the average PSA level, median Gleason score and median age results in metastatic and non-metastatic patients, revealing that the first two parameters were significantly higher in metastatic patients. However, there weren't $t$ any significant difference in age between the two categories.
TABLE 1: Median PSA level, Gleason score and median age in patients with positive and negative bone scans.

\begin{tabular}{lll}
\hline \hline & Positive; $\mathrm{n}=\mathbf{4 1}$ & Negative; $\mathrm{n}=\mathbf{4 3}$ \\
\hline Median PSA level (ng/ml) & 257.6 & 78.15 \\
Median Gleason score & 7.8 & 7.02 \\
Median age (years) & 70.7 & 71.9 \\
\hline \hline
\end{tabular}

$\mathrm{n}=$ number of patients

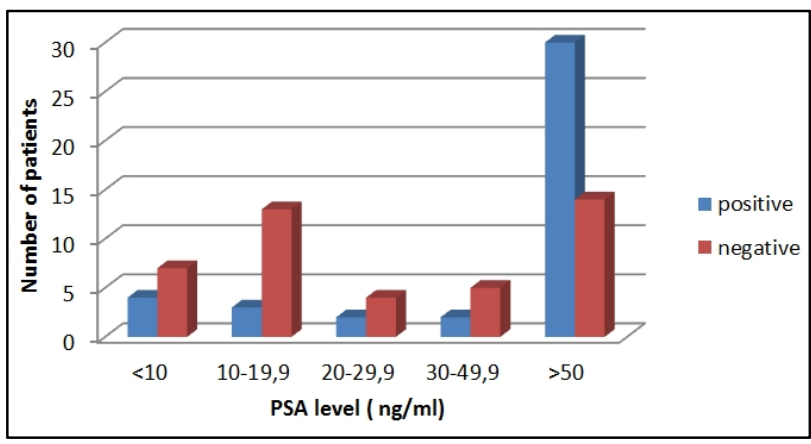

FIG. 5: Bone scan results in each PSA bracket.

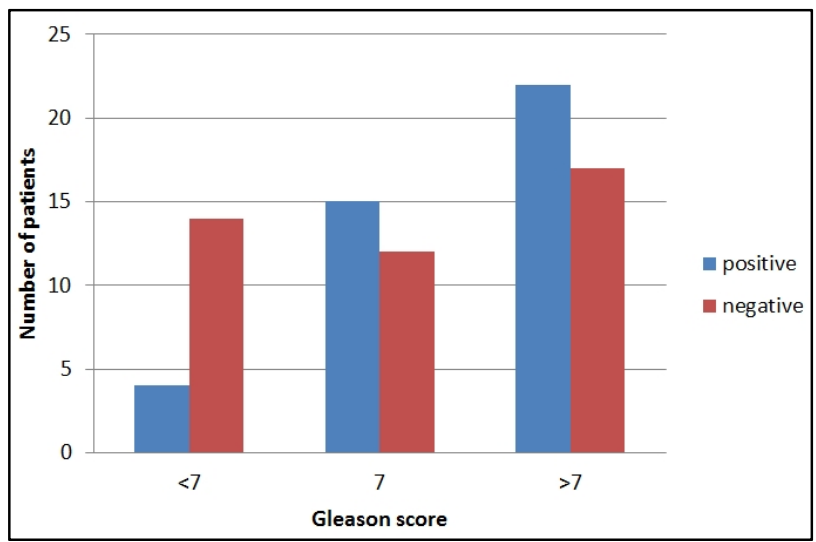

FIG. 6: Bone scan results in each Gleason bracket.

TABLE 2: Number of positive and negative bone scans in each PSA bracket.

\begin{tabular}{rccc}
\hline \multirow{2}{*}{$\begin{array}{c}\text { PSA level } \\
\text { (ng/ml) }\end{array}$} & \multicolumn{3}{c}{ Bone scan } \\
\cline { 2 - 4 }$<10$ & Positive & Negative & Total \\
\hline $10-19,9$ & 3 & 7 & 11 \\
$20-29,9$ & 2 & 13 & 16 \\
$30-49,9$ & 2 & 5 & 6 \\
$>50$ & 30 & 14 & 7 \\
Total & 41 & 43 & 84 \\
\hline \hline
\end{tabular}

PSA= Prostate specific antigen

TABLE 3: Number of positive and negative bone scans in each Gleason bracket.

\begin{tabular}{lccc}
\hline \hline Gleason & \multicolumn{3}{c}{ Bone scan } \\
\cline { 2 - 3 } \multicolumn{1}{c}{ score } & Positive & Negative & Total \\
\hline$<7$ & 4 & 14 & 18 \\
7 & 15 & 12 & 27 \\
$>7$ & 22 & 17 & 39 \\
Total & 41 & 43 & 84 \\
\hline \hline
\end{tabular}


TABLE 4: Number of positive bone scans with respect to PSA level and the Gleason score in asymptomatic patients.

\begin{tabular}{lll|l|l|ll}
\hline \hline & \multicolumn{7}{c}{ Gleason Score } \\
\cline { 2 - 7 } & & $<$ & \multicolumn{2}{c}{ 7 } & \multicolumn{2}{c}{$>$ 7 } \\
\cline { 2 - 7 } PSA(ng/ml) & $\mathrm{n}$ & BM+ & n & BM+ & n & BM+ \\
\hline$<10$ & 2 & 0 & 2 & 0 & 3 & 0 \\
$10-19,9$ & 5 & 0 & 3 & 0 & 3 & 0 \\
$20-29,9$ & 2 & 0 & 0 & 0 & 2 & 1 \\
$30-49,9$ & 2 & 1 & 3 & 0 & 2 & 1 \\
$\geq 50$ & 3 & 1 & 7 & 5 & 12 & 5 \\
\hline \hline
\end{tabular}

$\mathrm{BM}+$, positive bone metastases

According to Figure 5 and 6, Table 2 and 3, showing the relationship between PSA level, Gleason score and bone scan results, we concluded that the risk of occurrence of bone metastases increased with the elevation of PSA level or with Gleason score $\geq 7$. Hence, these two parameters are considered independent predictors of bone scan positivity.

Combining PSA level and Gleason score results in asymptomatic patients ( 51 patients) as shown in Table 4, we obtained the following results:

- $\quad$ None of the patients with no bony pain and with PSA level $<20 \mathrm{ng} / \mathrm{ml}$ had a positive bone scan irrespective of the Gleason score.

- $\quad$ None of the patients with no bony pain, with PSA level $<30 \mathrm{ng} / \mathrm{ml}$ and with a Gleason score < 7 had a positive bone scan.

\section{Discussion}

The pretreatment staging of prostate cancer directs therapeutic decision and provides important prognostic information. Skeleton is a common site of metastases, thus, early detection of bone and bone marrow metastases is essential for proper patient management. ${ }^{2}$

However, the detection of skeletal involvement in newly diagnosed prostate cancer is influenced by three major prognostic factors; PSA, clinical stage and Gleason Grade. ${ }^{7}$

The significant relationship between these parameters and the likelihood of positive bone scan has led to reduce a high number of potentially avoidable staging bone scan studies with a significant reduction of superfluous costs for the health care system. ${ }^{8}$

According to the European Association of Urology (EAU) guidelines, updated in April 2014, bone scan is recommended in asymptomatic patients only if the PSA level $>10 \mathrm{ng} / \mathrm{mL}$ or Gleason score $\geq 8$ or clinical stage $\geq$ T3 (intermediate-/high-risk situations). It should also be obtained in symptomatic patients, independently of the PSA level, Gleason score or clinical stage. ${ }^{6}$
Chybowski et al. ${ }^{9}$ had an experience with 521 patients with untreated newly diagnosed prostate cancer. They demonstrated that bone metastases did not occur in patients with PSA levels $<10 \mathrm{ng} / \mathrm{ml}$, but it did occur in 1 patient (1\%) with a PSA level of 10-19,9 $\mathrm{ng} / \mathrm{ml}$.

In another study performed by Gleave et al. ${ }^{10}$, scans were positive in none of the 290 patients with PSA levels below 10 $\mathrm{ng} / \mathrm{ml}, 4$ of $88(4.5 \%)$ with PSA levels between 10 and $19,9 \mathrm{ng} / \mathrm{ml}$.

Mcarthur et al. ${ }^{4}$, found in his large study including 672 patients that PSA level $<20 \mathrm{ng} / \mathrm{ml}$, combined with a Gleason score $<8$, had a negative predictive value for bone metastases of $100 \%$.

For Ritenour et al. ${ }^{3}$, bone scans can be omitted in asymptomatic patients with PSA $<10 \mathrm{ng} / \mathrm{ml}$ irrespective of the Gleason grade or in patients with Gleason score $\leq 7$ with PSA $\leq 30$ $\mathrm{ng} / \mathrm{ml}$.

In Asiatic population, several studies conducted by Miranda et al. ${ }^{11}$, Megumi et al. ${ }^{12}$, and Kosuda et al. ${ }^{13}$, concluded that bone scan might be avoidable in asymptomatic patients with PSA level $\leq 10 \mathrm{ng} / \mathrm{ml}$. A positive relation between the PSA level, Gleason score and presence of bone metastases on bone scan was demonstrated in our study, having a trend in line with the other studies.

Comparing the overall proportion of positive bone scans in this study to other previous studies, we found a substantial difference with higher rates of bone metastases: $49 \%$ vs. $4 \%$ in Ritenour et al. study ${ }^{3}, 8 \%$ in Mcarthur et al. study ${ }^{4}, 30 \%$ in Wymenga et al. study ${ }^{14}, 39,7 \%$ in Al-ghazo et al. study ${ }^{15}$.

This high rate of positive bone scans is partly due to the PSA screening programs weakly carried out in our country; therefore patients consult in a late stage of prostate cancer.

\section{Conclusion}

In the present study, we concluded that the PSA level and the Gleason score can be used to predict accurately the occurrence of bone involvement in patients with newly diagnosed prostate cancer. Moreover, we defined a group of patients in whom a baseline bone scan could be safely omitted: asymptomatic patients with PSA $<20 \mathrm{ng} / \mathrm{ml}$ irrespective of the Gleason grade, or patients with PSA level $<30 \mathrm{ng} / \mathrm{ml}$ and with a Gleason score $<7$.

By implementing the results of this study, bone scans could have been avoided in 30/84 patients allowing considerable cost savings and decreasing times from diagnosis to treatment. However, there are some limitations, mostly due to the low number of patients, the retrospective aspect of the study that may be prone to a possible selection bias. In addition, some 
other important criteria were not considered like clinical tumor stage and the tumor differentiation degree.

Therefore, the outcome of the study needs to be confirmed by a prospective cohort reviewing patients referred to Nuclear Medicine department for the pre-treatment staging of their newly diagnosed prostate cancer.

\section{Conflict of interest}

The authors declare that they have no conflicts of interest. The authors alone are responsible for the content and writing of the paper.

\section{References}

1. Beheshti M, Langsteger W, Fogelman I. Prostate cancer: role of SPECT and PET in imaging bone metastases. Semin Nucl Med. 2009;39:396-407.

2. Lebret T, Méjean A. Physiopathology, diagnosis and management of bone metastases from prostate cancer. Prog Urol. 2008;18 Suppl 7:S349-56.

3. Ritenour CW, Abbott JT, Goodman M, et al. The utilization of Gleason grade as the primary criterion for ordering nuclear bone scan in newly diagnosed prostate cancer patients. ScientificWorldJournal. 2009;9:1040-5.

4. McArthur C, McLaughlin G, Meddings RN. Changing the referral criteria for bone scan in newly diagnosed prostate cancer patients. $\mathrm{Br} \mathrm{J} \mathrm{Ra}-$ diol. 2012;85:390-4.

5. Zacho HD, Barsi T, Mortensen JC, et al. Prospective multicenter study of bone scintigraphy in consecutive patients with newly diagnosed prostate cancer. Clin Nucl Med. 2014;39:26-31.

6. Mottet N, Bastian PJ, Bellmunt J, et al. Guidelines on prostate cancer. European Association of Urology 2014. http://uroweb.org/wp-content/uploads/1607-Prosta te-Cancer_LRV3.pdf
7. Abuzallouf S, Dayes I, Lukka H. Baseline staging of newly diagnosed prostate cancer: a summary of the literature. J Urol. 2004;171:2122-7.

8. Briganti A, Passoni N, Ferrari M, et al. When to perform bone scan in patients with newly diagnosed prostate cancer: external validation of the currently available guidelines and proposal of a novel risk stratification tool. Eur Urol. 2010; 57:551-8.

9. Chybowski FM, Keller JJ, Bergstralh EJ, Oesterling JE. Predicting radionuclide bone scan findings in patients with newly diagnosed, untreated prostate cancer: prostate specific antigen is superior to all other clinical parameters. J Urol. 1991;145:313-8.

10. Gleave ME, Coupland D, Drachenberg D, et al. Ability of serum prostate-specific antigen levels to predict normal bone scans in patients with newly diagnosed prostate cancer. Urology 1996; 47: 708-12.

11. Lai MH, Luk WH, Chan JC. Predicting bone scan findings using sPSA in patients newly diagnosed of prostate cancer: feasibility in Asian population. Urol Oncol. 2011;29:275-9.

12. Hirobe M, Takahashi A, Hisasue S, et al. Bone scanning--who needs it among patients with newly diagnosed prostate cancer? Jpn J Clin Oncol. 2007; 37:788-92.

13. Kosuda S, Yoshimura I, Aizawa T, et al. Can initial prostate specific antigen determinations eliminate the need for bone scans in patients with newly diagnosed prostate carcinoma? A multicenter retrospective study in Japan. Cancer. 2002;94:964-72.

14. Wymenga LF, Boomsma JH, Groenier K, et al. Routine bone scans in patients with prostate cancer related to serum prostate-specific antigen and alkaline phosphatase. BJU Int. 2001;88:226-30.

15. Al-Ghazo MA, Ghalayini IF, Al-Azab RS, et al. Do all patients with newly diagnosed prostate cancer need staging radionuclide bone scan? A retrospective study. Int Braz J Urol. 2010; 36:685-91; discussion 691-2. 